\title{
Frecuencia y caracterización del trauma de tórax. Cuenca - Ecuador
}

\section{Frequency and characterization of chest trauma. Cuenca - Ecuador}

\author{
Jorge Eduardo Ávila Narváez*1, Juan Andrés Ávila Narváez ${ }^{1}$, Jessica Gabriel Vásquez Cevallos ${ }^{2}$, Lisette \\ Espinosa Martín ${ }^{3}$, Edison Gustavo Moyano Brito ${ }^{3}$ \\ ${ }^{1}$ Hospital General de Quevedo, Quevedo, Los Ríos, Ecuador \\ 2 Hospital Homero Castanier, Azogues, Ecuador \\ ${ }^{3}$ Universidad Católica de Cuenca, Ecuador \\ *edward_avnar89@hotmail.com
}

DOI: https://doi.org/10.26871/killkanasalud.v4i4.749

\begin{abstract}
Resumen
Antecedentes: el traumatismo de tórax es una patología altamente desafiante debido a que existe compromiso de órganos vitales, con la elevada posibilidad de falla multiorgánica y muerte. Objetivo: determinar la frecuencia y la severidad del trauma de tórax en pacientes atendidos en el Hospital Vicente Corral Moscoso y José Carrasco Arteaga, periodo 2018. Materiales y métodos: se trata de un estudio descriptivo transversal. La muestra fueron 248 pacientes con trauma de tórax que cumplieron los criterios de inclusión. Los datos se recolectaron mediante un formulario diseñado por el autor. La estadística descriptiva se presentó con las medidas de centralización (media) y dispersión (DS) para las variables cuantitativas, y para las cualitativas con valores de frecuencia y porcentaje. La información se tabuló en el software estadístico SPSS v. 15. Resultados: la frecuencia trauma de tórax fue del 17,2\%; el promedio de edad del $42,03 \pm$ DS 18,74 años; el $60 \%$ pacientes fueron masculinos; además, el $30 \%$ tenían un trauma toracoabdominal; el $20 \%$ la etiología fue accidente laboral; y el $80 \%$ tuvieron un trauma cerrado y el $50 \%$ de ellos fue directo. En los traumas abiertos, el $20 \%$ fue por arma blanca. El $20 \%$ de las complicaciones fueron por neumotórax, enfisema y hemitórax; el $60 \%$ tuvieron un manejo quirúrgico, y de estos, los tipos de intervenciones más frecuentes fueron el drenaje pleural; y la severidad fueron leves en el $60 \%$. Conclusiones: el trauma de tórax fue frecuente en el sexo masculino, la etiología por accidente laboral, siendo el neumotórax la complicación más usual.
\end{abstract}

Palabras clave: trauma de tórax, severidad, frecuencia, hemotórax, neumotórax .

\begin{abstract}
Background: chest trauma is a highly challenging pathology due to the involvement of vital organs, with the high possibility of multi-organ failure and death. Objective: to determine the frequency and severity of chest trauma in patients treated at the Vicente Corral Moscoso and José Carrasco Arteaga Hospital, period 2018. Materials and methods: this is a descriptive cross-sectional study. The sample consisted of 248 patients with chest trauma who met the inclusion criteria. The data were collected using a form designed by the author. Descriptive statistics were presented with measures of centralization (mean) and dispersion (SD) for quantitative variables, and for qualitative variables with frequency and percentage values. The information was tabulated in the statistical software SPSS v. 15. Results: chest trauma frequency was 17.2\%; the average age of $42.03 \pm$ SD 18.74 years; $60 \%$ patients were male; furthermore, $30 \%$ had a thoracoabdominal trauma; $20 \%$ the etiology was work accident; and $80 \%$ had a blunt trauma and 50\% of them was direct. In open trauma, 20\% was caused by a knife. $20 \%$ of the complications were due to pneumothorax, emphysema and hemithorax; $60 \%$ had a surgical management, and of these, the most frequent types of interventions were pleural drainage; and the severity were mild in $60 \%$. Conclusions: chest trauma was frequent in males, the etiology was due to occupational accident, pneumothorax being the most common complication.
\end{abstract}

Keywords: chest trauma, severity, frequency, hemothorax, pneumothorax.

\section{Introducción}

El trauma presenta tres picos de mortalidad: el inicial o primario que se presenta durante los primeros minutos y aporta con un $50 \%$ de probabilidades de fallecimiento, el segundo en las 3 horas siguientes y contribuye con un $20 \%$ y el tercero relacionado con la mortalidad tardía con 
un $30 \%$. Por ello, se ha considerado que la primera hora luego del traumatismo como el "periodo de oro", durante el cual un manejo adecuado, rápido y oportuno nos otorga mayores posibilidades de prolongar la vida del paciente. ${ }^{1}$

El traumatismo torácico es la segunda lesión no intencional más prevalente en los Estados Unidos y se asocia con una morbilidad significativa, En particular, las fracturas de costillas ocurren en hasta el $10 \%$ de pacientes con traumatismos hospitalizados y pueden estar asociados con una mortalidad de $3 \%$ a $13 \% .^{2}$

Constituye una causa frecuente de mortalidad temprana y tardía en el paciente severamente traumatizado. La presentación, detección precoz y por supuesto una intervención adecuada, influyen directamente en el desenlace del paciente. Un alto porcentaje de personas víctimas de trauma severo desarrollarán un trauma torácico, y a su vez los pacientes cuya fuente primaria de muerte no fue recocida clínicamente presentarán dicha patología. ${ }^{3}$

El 25-50\% de los fallecimientos atribuidos a las lesiones torácicas tienen una pueden estar asociadas a una serie de causas que incluyen: caídas, accidentes de tráfico, lesiones deportivas y agresiones físicas y se especifican varias formas de presentación clínica de los traumas de tórax entre los que se describen: hematomas simples y fracturas costales, hemotórax traumático o neumotórax asociado con daño orgánico interno por fracturas costales; en casos extremos, se produce un traumatismo torácico abierto con exposición de la cavidad torácica ${ }^{4}{ }^{5}$

El proceso de priorizar a los pacientes sobre la base de la agudeza clínica, es un principio clave en el manejo efectivo de un incidente importante. La efectividad general del proceso de triage no es solo un equilibrio entre la identificación de aquellos que necesitan o no una intervención para salvar vidas, sino también aquellos a quienes se subestimó o se les tildó incorrectamente de requerirla o no. ${ }^{6}$

La mayoría de los pacientes con trauma torácico se puede manejar de forma conservadora solo con el drenaje del tubo torácico. Sin embargo, una pequeña minoría requiere una toracotomía en el departamento de urgencias, también denominada "toracotomía de resucitación". Este procedimiento está indicado en casos de lesiones torácicas aisladas que ponen en peligro la vida, o como parte de la RCP (resucitación cardiopulmonar) en pacientes con traumatismo múltiple, o en pacientes con traumatismo torácico con sangrado masivo de otras lesiones. ${ }^{3}$

El trauma torácico sigue siendo una lesión relevante para el paciente politraumatizado. ${ }^{8} \mathrm{El} 50 \%$ de todos los traumas, representan los traumatismos torácicos ${ }^{4}$ por lo cual esta patología representa hoy en día una de las causas más importantes de mortalidad y morbilidad alrededor del mundo 5.6

En Ecuador, según el Servicio de Investigación de Accidentes de Tránsito (SIAT) en un $90 \%$ son de causa humana (conductores, peatones y pasajeros), el $5 \%$ provocado por daños en las vías y carreteras y los demás provienen de otros problemas. ${ }^{1}$
La Provincia del Guayas registro en el período 2004 - 2007 un promedio de 5.051 accidentes de tránsito por año de los cuáles se obtuvieron 169 lesionados por caídas desde los transportes públicos, 933 por arrollamiento; el común denominador del accidente fue la imprudencia del conductor por encontrarse en estado etílico, 435 murieron, 1060 resultaron heridos y 11 sufrieron traumas severos. El común denominador del accidente fue la imprudencia del conductor por encontrarse en estado etílico. ${ }^{12}$

La National Library of Medicine, informaron que la muerte por trauma de tórax oscila entre el 20 al $25 \%$ en pacientes politraumatizados, concluyendo además que su prevalencia fue de $4.57 \%$ en el servicio de emergencias, donde $72 \%$ lo sufrieron los hombres y $28 \%$ a mujeres, con una edad promedio de 40.8 años. ${ }^{7}$

Los traumatismos torácicos representan la sexta causa de muerte y la quinta en discapacidad significativa a nivel mundial. Resultando alrededor de 5 millones de decesos anualmente por esta patología en poblaciones menores de 65 años. ${ }^{8}$

Según la ATLS (Advanced Traume Life Suport), el trauma de tórax se produce por acciones de fuerza extrema que lesiona estructuras óseas y órganos internos del tórax, poniendo en riesgo la función de los órganos y comprometiendo la vida del paciente. En función a que exista o no una solución de continuidad de la pared torácica, se ha clasificado a esta patología en trauma cerrado y abierto, representando el $70 \%$ y el $30 \%$ respectivamente. ${ }^{8}$

Se ha documentado que los traumatismos torácicos tienen diversas causas, estimándose que por lo general el $80 \%$ de ellos son producto de accidentes de tránsito seguidos de los laborales, ciertas prácticas deportivas, agresiones, caídas, suicidio, entre otras ${ }^{8} .{ }^{9}$ Las lesiones asociadas al traumatismo torácico son: hemotórax $(65.3 \%)$, hemo neumotórax $(10.7 \%)$, contusión pulmonar $(4 \%)$, neumotórax con enfisema (4\%). ${ }^{9}$

El traumatismo torácico se asocia con alteración de la inflamación postraumática, mayores tasas de complicación y resultados alterados en estos pacientes críticos, por lo tanto, el tratamiento correcto y estandarizado sigue siendo un reto. Con el fin de reducir las complicaciones respiratorias secundarias como la neumonía y el síndrome de dificultad respiratoria aguda (SDRA). ${ }^{10}$

Las muertes producidas dentro de las 3 horas del accidente con lesión torácica suelen ser resultado de obstrucción de vía aérea, alteraciones ventilatorias, hemorragias incontrolables o taponamiento cardiaco. Cerca del $85 \%$ de pacientes con trauma de tórax pueden manejarse con medidas menores como: drenaje pleural, soporte ventilatorio, control del dolor y observación, del 15 al $30 \%$ de las lesiones penetrantes requerirá toracotomía. ${ }^{11}$

En EE.UU. El traumatismo estar considerado como la tercera causa de mortalidad en pacientes con edades entre los 33 a 44 años de edad, lo cual aproximadamente representa el $25 \%$ por 100.000 muertes de esta causa, lo que 
equivale a 1.2 millones de personas y deja alrededor de 50 millones de personas heridas o discapacitadas ${ }^{3} .{ }^{17}$

El costo aproximado destinado por el Banco Mundial para los países en vías de desarrollo en lo que se refiere a los accidentes de tránsito es de aproximadamente $1 \%$ del Producto Interno Bruto. En lo que se refiere a nuestro país, en el año 2007, se consideró la primera causa de muerte en pacientes menores que tienen entre 5 a 14 años de edad, con un promedio de 50 personas fallecidas por cada mes; lo que significa un desembolso aproximado de doscientos millones de dólares por año ${ }^{6} .^{18}$

La Provincia del Guayas registro en el período 2004 - 2007 un promedio de 5.051 accidentes de tránsito por año de los cuáles se obtuvieron 169 lesionados por caídas desde los transportes públicos, 933 por arrollamiento; el común denominador del accidente fue la imprudencia del conductor por encontrarse en estado etílico, 435 murieron, 1060 resultaron heridos y 11 sufrieron traumas severos. El común denominador del accidente fue la imprudencia del conductor por encontrarse en estado etílico ${ }^{3} .{ }^{19}$

\section{Metodología}

Tipo de estudio: descriptivo transversal.

Población y muestra de estudio: todos los pacientes de ambos sexos desde los 18 años con diagnóstico de trauma de tórax en el servicio de emergencia del Hospital Vicente Corral Moscoso y el Hospital José Carrasco Arteaga durante el periodo enero-diciembre 2018.

Procedimientos: se solicitó autorización para la realización del estudio a los directores del HVCM y del HJCA. Se elaboró un cuestionario de recolección de datos con las variables de estudio el mismo que tuvo 2 secciones: la primera de variables sociodemográficas de la población de estudio y la segunda sección incluyó preguntas orientadas a determinar la información concerniente al trauma de tórax y la escala de severidad del mismo. Para medir la severidad del trauma de tórax se utilizó la escala de El Thorax Trauma Severity Score (TTSS) fue desarrollado para la evaluación temprana del traumatismo torácico e identificación de pacientes con alto riesgo de complicaciones en una población de traumatismo grave. La escala evalúa la edad del paciente, el índice $\mathrm{PaO} 2 / \mathrm{FiO} 2$, el número de fracturas costales, la contusión pulmonar y la presencia de hemo/neumotórax.

Plan de tabulación y análisis de resultados: para poder conocer y detallar la tabulación de datos y los análisis, se presentaron los datos en forma de tablas. En dependencia del tipo de variable con el propósito de resumir la información se trabajó: cuantitativas con la media aritmética (x) y la desviación estándar (DS) para variables cualitativas con frecuencias $\left(\mathrm{N}^{\circ}{ }^{\circ}\right.$ ) y porcentajes. De igual manera en el manejo, la presentación y el análisis de datos se utilizaron los programas IBM SPSS 22.0 y Epi Info 07 y Epidat 4.1.

Procedimientos bioéticos: previo a la ejecución del estudio, se presentó protocolo y fue revisado y aprobado por el Comité de Ética. El manejo de los datos recolectados fue confidencial, ya que en el formulario no se mencionó nombres, únicamente se trabajó con números de historias clínicas y a cada una de estas se le asigno un código de referencia, además, el manejo de la base de datos se usó únicamente con fines investigativos, de esta manera se garantizó la confidencialidad de los participantes. Los beneficios son múltiples, ya que al conocer las características nos ayuda para establecer un mejor manejo a los pacientes con estas lesiones. Debido a que la información se recolectó de las historias clínicas no requirió consentimiento informado. El acceso a la información recolectada está a cargo del investigador del estudio, pero, además, se indica que se encuentra disponible, en caso de ser requerida por el Comité de Bioética de la Universidad de Cuenca. Los registros de papel se mantendrán en un lugar cerrado y protegido luego de la presentación y respectiva defensa de la tesis.

En la realización de este estudio, declaramos que no existen conflictos de interés.

\section{Resultados}

Se realizó el estudio en 248 pacientes con trauma de tórax, tomando como prueba evidente sus historias clínicas, de las cuáles 150 historias clínicas fueron tomados del Hospital Vicente Corral Moscoso, y 98 historias clínicas del Hospital José Carrasco Arteaga.

Tabla 1: Características sociodemográficas de los 248 pacientes con trauma de tórax de los hospitales Vicente Corral Moscoso y José Carrasco Arteaga, periodo 2018

\begin{tabular}{lcc}
\hline Características sociodemográficas & $\mathrm{n}(248)$ & $\%$ \\
\hline Edad & & \\
\hline 18 a 35 años & 114 & 46,0 \\
36 a 64 años & 94 & 37,9 \\
65 o más años & 40 & 16,1 \\
\hline Sexo & & \\
\hline Masculino & 147 & 59,3 \\
Femenino & 101 & 40,7 \\
\hline Residencia & & \\
\hline Urbana & 160 & 64,5 \\
Rural & 88 & 35,5 \\
\hline
\end{tabular}

La media de edad de 42,03 \pm DS 18,74; con una mínima de edad de 18 años y una máxima de 94 años. En relación al sexo, 6 de cada 10 pacientes con trauma de tórax fueron masculinos, e igualmente 6 de cada 10 de residencia urbana (Tabla 1). 
Tabla 2: Características del trauma de tórax de los 248 pacientes de los hospitales Vicente Corral Moscoso y José Carrasco Arteaga, periodo 2018

\begin{tabular}{|c|c|c|}
\hline Caracterización del Trauma de tórax & $\mathrm{n}$ & $\%$ \\
\hline \multicolumn{3}{|l|}{ Extensión del Trauma } \\
\hline Torácico Puro & 37 & 14,9 \\
\hline Cérvico Torácico & 43 & 17,3 \\
\hline Toracoabdominal & 64 & 25,8 \\
\hline Tórax y extensiones & 55 & 22,2 \\
\hline Politraumatismos & 49 & 19,8 \\
\hline Accidentes de transito & 52 & 21,0 \\
\hline Caídas & 61 & 24,6 \\
\hline \multicolumn{3}{|l|}{ Etiología del trauma } \\
\hline Accidentes Laborales & 52 & 21,0 \\
\hline Arrollamiento o aplastamiento & 22 & 8,9 \\
\hline Otros & 61 & 24,6 \\
\hline \multicolumn{3}{|l|}{ Mecanismo del trauma } \\
\hline Abierto & 43 & 17,3 \\
\hline Cerrado & 205 & 82,7 \\
\hline \multicolumn{3}{|l|}{ Mecanismo de trauma de tórax abierto } \\
\hline Arma blanca & 32 & 12,9 \\
\hline Arma de fuego & 11 & 4,4 \\
\hline Otro & 205 & 82,7 \\
\hline \multicolumn{3}{|l|}{ Mecanismo de trauma de tórax cerrado } \\
\hline Directo & 139 & 56,0 \\
\hline Indirecto & 65 & 26,2 \\
\hline Otro & 44 & 17,8 \\
\hline \multicolumn{3}{|l|}{ Complicaciones } \\
\hline Hemitórax & 82 & 20,9 \\
\hline Hemoneumotórax & 66 & 16,8 \\
\hline Fracturas costales & 60 & 15,3 \\
\hline Enfisema & 79 & 20,2 \\
\hline Lesión Cardiaca & 13 & 3,1 \\
\hline Neumotórax & 91 & 23,3 \\
\hline \multicolumn{3}{|l|}{ Tipo de lesión en parénquima } \\
\hline Contusión Pulmonar & 112 & 45,2 \\
\hline Laceración Pulmonar & 6 & 2,4 \\
\hline Quiste Trauma & 1 & 0,4 \\
\hline Hematoma Pulmonar & 8 & 3,2 \\
\hline Ninguno & 121 & 48,8 \\
\hline
\end{tabular}

La frecuencia del trauma de tórax fue del $17,2 \%$, y en general, los pacientes presentaron complicaciones mixtas; por lo cual, 3 de cada 10 tenían un trauma toracoabdominal, seguidos del trauma de tórax por caída; en relación a la etiología, el 2 de cada 10 fueron por accidentes laborales; y por cada 10 pacientes, 8 tuvieron un trauma cerrado, y 5 de ellos fue directo. En los traumas abiertos, 2 de cada 10 fue por arma blanca. Las complicaciones más frecuentes del traumatismo de tórax fue el neumotórax, seguido del enfisema y hemitórax, esto en similar frecuencia (2 por cada 10 traumatismos torácicos) (Tabla 2).

Tabla 3: Características quirúrgicas del trauma de tórax de los 248 pacientes de los hospitales Vicente Corral Moscoso y José Carrasco Arteaga, periodo 2018

\begin{tabular}{lcc}
\hline Manejo Quirúrgico & $\mathrm{n}$ & $\%$ \\
\hline $\mathrm{Si}$ & 139 & 56,0 \\
$\mathrm{No}$ & 109 & 44,0 \\
\hline Tipo de Cirugía & & \\
\hline Drenaje Pleural & 223 & 82,2 \\
Toracotomía & 1 & 0,36 \\
Laparotomía & 8 & 2,9 \\
V.A.T.S. & 40 & 14,7 \\
\hline
\end{tabular}

Tabla 3: Características quirúrgicas del trauma de tórax de los 248 pacientes de los hospitales Vicente Corral Moscoso y José Carrasco Arteaga, periodo 2018... (continuación)

\begin{tabular}{lcc}
\hline Complicaciones Posquirúrgicas & & \\
\hline Atelectasias & 16 & 6,5 \\
Neumotórax Persistente & 6 & 2,4 \\
Síndrome de ocupación pleural & 8 & 3,2 \\
Empiema & 7 & 2,8 \\
Ninguna & 211 & 65,1 \\
\hline Ingreso a UCI & & \\
\hline Si & 7 & 2,8 \\
No & 241 & 97,2 \\
\hline
\end{tabular}

De los pacientes que conformaron el estudio, la mayoría tuvieron de 1 a 2 resoluciones quirúrgicas; por lo que, por cada 10 paciente, 6 tuvieron un manejo quirúrgico; y de estos, los tipos de intervenciones más frecuentes fueron el drenaje pleural, seguido del video torascoscopia asistida; además, en la mayoría el tratamiento fue el adecuado, sin tener la necesidad de estar los pacientes ingresados en cuidados intensivos (Tabla 3 ).

Tabla 4: Severidad del trauma de tórax de los 248 pacientes de los hospitales Vicente Corral Moscoso y José Carrasco Arteaga, periodo 2018

\begin{tabular}{lcc}
\hline Variable & Frecuencia & Porcentaje \\
\hline Leve & 144 & 58,1 \\
Moderado & 88 & 35,5 \\
Grave & 16 & 6,5 \\
Total & 248 & 100,0 \\
\hline
\end{tabular}

Para realizar el índice de severidad de trauma de tórax, se tomó en cuenta la edad, fracturas costales, contusión y lesiones de la pleura. En relación a la severidad del trauma de toras, por cada 10 pacientes 6 fueron leves segundo del moderado (Tabla 4).

\section{Discusión}

En esta investigación, se encontró una media de edad de 42,03 \pm DS 18,74; con una mínima de edad de 18 años y una máxima de 94 años. En relación al sexo, 6 de cada 10 pacientes con trauma de tórax fueron masculinos, e igualmente 6 de cada 10 de residencia urbana (Tabla 1). Datos que se comparan con el estudio de Almed N, y cols., ${ }^{20}$ donde la mayoría fue del sexo masculino en el $46 \%$ de los casos, comparado con el 44,2\% de mujeres que tenían un antecedente de trauma torácico. A la vez, SchulzDrost S., y cols., , ${ }^{21}$ en el año 2019 en Alemania, realizó un análisis retrospectivo del conjunto de datos TraumaRegister DGU, donde incluyeron un total de 49.567 pacientes; donde el $72,7 \%$ fueron varones. Larrea M., ${ }^{22}$ en el año 2009 encontró igualmente que esta lesión predomina más en el sexo masculino que en el femenino, en una relación de 9:1.

En este estudio la mayoría de los pacientes presentó complicaciones mixtas que se tomaron en cuenta para la caracterización; por lo cual, 3 de cada 10 tenían un trauma toracoabdominal, seguidos del trauma de tórax por caída; 
en relación a la etiología, el 2 de cada 10 fueron por accidentes laborales; y por cada 10 pacientes, 8 tuvieron un trauma cerrado, y 5 de ellos fue directo. En los traumas abiertos, 2 de cada 10 fue por arma blanca. Las complicaciones más frecuentes del traumatismo de tórax fue el neumotórax, seguido del enfisema y hemitórax, esto en similar frecuencia (2 por cada 10 traumatismos torácicos) (Tabla 2). Resultados que se comparan con Schulz-Drost S., y cols., ${ }^{21}$ donde el $95.9 \%$ tenían un trauma torácico cerrado, y $14.008(28,3 \%)$ tenían contusiones pulmonares graves. A diferencia en cambio con Liebsch C.,,${ }^{23}$ donde las fracturas de costilla representaron la complicación más común, que ocurre en el $10-20 \%$ de todos los pacientes con traumatismo torácico cerrado y conduce a lesiones concomitantes de los órganos internos en casos severos. Por ello, en Alemania, en total, se identificaron 3.735 fracturas de costillas (9,8 por paciente). El $54 \%$ de las fracturas de costillas se detectaron en el tórax izquierdo. En promedio, la mayoría de las fracturas de costillas ocurrieron en lesiones por aplastamiento / enterramiento $(15,8, \mathrm{n} 13)$ $\mathrm{y}$ accidentes de peatones $(12,8, \mathrm{n} 13)$, menos en accidentes automovilísticos / de camiones $(8,9, \mathrm{n} 75)$.

En los pacientes de este estudio, la mayoría tuvo 1 o 2 resoluciones quirúrgicas; por lo que, por cada 10 paciente, 6 tuvieron un manejo quirúrgico; y de estos, los tipos de intervenciones más frecuentes fueron el drenaje pleural, seguido del video torascoscopia asistida; además, en la mayoría el tratamiento fue el adecuado, sin tener la necesidad de estar los pacientes ingresados en cuidados intensivos (Tabla 3). En cambio, Almed N, y cols., , ${ }^{20}$ en el año 2019, accedieron al conjunto de datos del Banco Nacional de Datos de Trauma (NTDB) de 2012-2014 para el estudio. Todos los pacientes, de 65 años de edad y mayores, que experimentaron una caída y sufrieron una lesión torácica, fueron incluidos en el estudio. De los 15,256 pacientes que cumplieron con los criterios de inclusión, aproximadamente el $52 \%$ (7994) de los pacientes fueron tratados en una institución de atención de nivel superior. Concluyeron que el tratamiento de pacientes de edad avanzada con traumatismo torácico cerrado en instituciones de atención de nivel superior no mostró ningún beneficio en la supervivencia general o la duración de la estancia hospitalaria.

En relación a la severidad del trauma de tórax, por cada 10 pacientes 6 fueron leves segundo del moderado (Tabla 4). Resultados que difieren de Leijdesdorff HA, y cols., ${ }^{23}$ en el año 2020, quienes analizaron un total de 497 víctimas fallecidas, de las cuales la mayoría eran ciclistas. El trauma del tórax fue más frecuente en motociclistas y automovilistas $(60,9 \%$ y $65,8 \%$, respectivamente). Las víctimas menores de 25 años sufrieron lesiones más graves (mediana 38, IC95 \% 29-46). Más del $10 \%$ de las víctimas fueron gravemente lesionadas y no fueron transportadas a un centro de trauma de nivel I. La mayoría de este grupo tenía más de 75 años. Schulz-Drost S., y cols., ${ }^{21}$ reportó que para la contusión pulmonar severa que fue la más frecuente, la edad promedio fue $(44.7 \pm 19.7$ años $)$ y la intubación, cuidados intensivos, falla (multi-) orgánica, sepsis y lesiones extratorácicas fueron más comunes en este grupo. Los pacientes más jóvenes mostraron una mayor incidencia de CP que los mayores; sin embargo, la alta edad del paciente fue un factor de riesgo altamente significativo para el desarrollo de complicaciones y un mal resultado.

Las limitaciones de este estudio fue el describir el mecanismo de transporte, y la estancia hospitalaria, no se hizo análisis relacional. Sin embargo, coinciden que el sexo masculino es el más afectado y las complicaciones aumentan según se incrementa la edad de los pacientes.

\section{Conclusiones}

Las lesiones torácicas fueron predominantes en el sexo masculino, con una relación 2:1. El promedio de edad fue de 41 años en ambos sexos.

Las lesiones más frecuentes según la cinemática del trauma fueron accidentes laborales y caídas sin embargo en menor cantidad, pero no menos importantes fueron los traumatismos abiertos siendo más por arma blanca.

Según la clasificación CIE- 10, las lesiones predominantes fueron: Neumotórax, Hemo neumotórax Fracturas costales Enfisema, Lesiones cardiacas

El trauma abierto se presentó con mayor frecuencia que el trauma cerrado. El promedio de días de hospitalización por trauma torácico fue de 3 días.

El trauma cerrado fue más frecuente en el grupo de cinemática clasificada como caídas y accidentes de tránsito.

El trauma abierto más frecuente en el grupo de cinemática clasificada como riña por arma blanca.

Solo una persona falleció. El paciente con el desenlace desfavorable, respondía a un trauma abierto, por arma blanca con APACHE mayor de 7, hemo neumotórax masivo.

\section{Fuente de Financiamiento}

Este estudio es autofinanciado.

\section{Conflicto de Intereses}

No existen conflictos personales, profesionales, financieroso de otro tipo.

\section{Consentimiento Informado}

Los autores cuentan con el consentimiento informado de los pacientes para la investigación, la publicación del caso y sus imágenes.

\section{Referencias Bibliográficas}

1. Rodriguez R, Ferrero L, Escalona M, Garcias L. Reintervenciones quirúrgicas en pacientes con traumatismo torácico, abdominal o ambos. Mediciencias UTA. 2017;1(1):1-6. doi: https://doi.org/10.31243/mdc. uta.v1i1.7.2017

2. Galvagno SM, Smith CE, Varon AJ, Hasenboehler EA, Sultan S, Shaefer G, et al. Pain management for blunt thoracic trauma: A joint practice management guideline 
from the Eastern Association for the Surgery of Trauma and Trauma Anesthesiology Society. J Trauma Acute Care Surg. noviembre de 2016;81(5):936-51.

3. Unsworth A, Curtis K, Asha SE. Treatments for blunt chest trauma and their impact on patient outcomes 1 and health service delivery. Scand J Trauma Resusc Emerg Med 2015 Feb 8;23:17. doi: 10.1186/s13049015-0091-5. Disponible en: https://www.ncbi.nlm.nih. gov/pmc/articles/PMC4322452/

4. Ekpe EE, Eyo C. Determinants of mortality in chest 16 . trauma patients. Niger J Surg. 2014;20(1):30-34. doi:10.4103/1117-6806.127107

5. Hwang EG, Lee Y. Effectiveness of intercostal nerve block for management of pain in rib fracture patients. J Exerc Rehabil. 31 de agosto de 2014;10(4):241-4.

6. Vassallo JM, Smith JE, Wallis LA. Investigating the effects of under-triage by existing major incident triage tools. Eur J Emerg Med. 2019 Apr;26(2):139-144. doi: 18. 10.1097/MEJ.0000000000000513.

7. Refaely Y, Koyfman L, Friger M, Ruderman L, Abu Saleh M, Klein M, et al. Predictors of survival after emergency 19 department thoracotomy in trauma patients with predominant thoracic injuries in Southern Israel: a retrospective survey. Open Access Emerg Med. abril de 2019; Volume 20. 11:95-101.

8. Horst K, Andruszkow H, Weber CD, Pishnamaz M, Herren $\mathrm{C}$, Zhi Q, et al. Thoracic trauma now and then: A 10 year experience from 16,773 severely injured patients. Puebla I, editor. PLOS ONE. 19 de octubre de 21 2017;12(10):e0186712.

9. Al-Koudmani I, Darwish B, Al-Kateb K, Taifour Y. Chest trauma experience over eleven-year period at al-mouassat university teaching hospital-Damascus: a retrospective review of 888 cases. J Cardiothorac Surg. 19 de abril de 22 2012;7:35.

10. Yadollahi M, Paydar S, Sabetianfard Jahromi G, Khalili H, Etemadi S, Abbasi H, et al. Types and Causa- 23. lities in Dead Patients Due to Traumatic Injuries. Arch Trauma Res. 2015;4(1):e26028. Published 2015 Mar 20. doi:10.5812/atr.26028

11. Alborzi Z, Zangouri V, Paydar S, Ghahramani Z, Shafa M, Ziaeian B, et al. Diagnosing Myocardial Contusion after Blunt Chest Trauma. J Tehran Univ Heart Cent. 13 de abril de 2016;11(2):49-54.

12. Akoglu H, Celik OF, Celik A, Ergelen R, Onur O, Denizbasi A. Diagnostic accuracy of the Extended Focused Abdominal Sonography for Trauma (E-FAST) performed by emergency physicians compared to computed tomography. Am J Emerg Med. 2018 Jun;36(6):1014-1017. doi: 10.1016/j.ajem.2017.11.019. Epub 2017 Nov 8.

13. Avila M, Jenit R. Fractura costal como factor asociado para contusion miocardiaca en pacientes con trauma toracico cerrado atendidos en el hospital regional docente de trujillo. 2017; TESIS. disponible en: http://repositorio. upao.edu.pe/handle/upaorep/2663

14. Chien C-Y, Chen Y-H, Han S-T, Blaney GN, Huang T-
$\mathrm{S}$, Chen K-F. The number of displaced rib fractures is more predictive for complications in chest trauma patients. Scand J Trauma Resusc Emerg Med. 2017 Feb 28;25(1):19. doi: 10.1186/s13049-017-0368-y.

. Wutzler S, Sturm K, Lustenberger T, Wyen H, Zacharowksi K, Marzi I, et al. Kinetic therapy in multiple trauma patients with severe thoracic trauma: a treatment option to reduce ventilator time and improve outcome. Eur $\mathbf{J}$ Trauma Emerg Surg. 1 de abril de 2017;43(2):155-61.

Mendoza Rodríguez M, Tacuba JLA, Verdiguel DG, Valerio RH, López González A. Comportamiento epidemiológico del traumatismo torácico en las unidades de cuidados intensivos de hospitales de trauma. Med Crítica. 2014;28(3):164-74.

17. Coordinación General de Desarrollo Estratégico en Salud Dirección de Inteligencia de la Salud, Ministerio de Salud Pública. MSP; 2013.

8. González Fernández AM, Ramón Torres A, Valverde Molina J. Traumatismo torácico, neumotórax, hemoptisis y tromboembolismo pulmonar. Neumoped. 2017:189-209

9. Feliciano DV. Cardiac, Great Vessel, and Pulmonary Injuries. En: Rich's Vascular Trauma [Internet]. Elsevier; 2016 [citado 26 de mayo de 2019]. p. 71-99.

0. Ahmed N, Kuo YH. Evaluating the outcomes of blunt thoracic trauma in elderly patients following a fall from a ground level: higher level care institution vs. lower level care institution. Eur J Trauma Emerg Surg. 2019; https: //doi.org/10.1007/s00068-019-01230-1

. Schulz-Drost S, Finkbeiner R, Lefering R, Grosso M, Krinner S, Langenbach A, et al. Lung Contusion in Polytrauma: An Analysis of the TraumaRegister DGU. Thorac Cardiovasc Surg. 2019 Dec 13; DOI: 10.1055/s-00391700505

. Liebsch C, Seiffert T, Vlcek M, Beer M, Huber-Lang M, Wilke HJ. Patterns of serial rib fractures after blunt chest trauma: An analysis of 380 cases. PLoS One. 2019;14(12).

3. Leijdesdorff HA, Gillissen S, Schipper IB, Krijnen P. Injury Pattern and Injury Severity of In-Hospital Deceased Road Traffic Accident Victims in The Netherlands: Dutch Road Traffic Accidents Fatalities. World J Surg. 2020; Doi: 10.1007/s00268-019-05348-6

Recibido: 11 de octubre de 2020

Aceptado: 25 de octubre de 2020 